\title{
Making MLSD-decisions on Some Individual Symbols in a Sequence by Thresholding the Matched Filter Output
}

\author{
Per Ödling ${ }^{1}$ \\ Håkan B. Eriksson ${ }^{1,2}$ \\ Per Ola Börjesson ${ }^{1}$ \\ ${ }^{1}$ Luleå University of Technology, Division of Signal Processing, S-971 87 Luleå, Sweden \\ ${ }^{2}$ Ericsson Mobile Communications AB, Advanced Studies, S-223 70 Lund, Sweden
}

\begin{abstract}
We present a detector that, by thresholding the output of the matched filter, gives MLSDdecisions on binary, antipodally modulated symbols that have been corrupted by intersymbol interference and additive Gaussian noise. The detector is simple in structure, consisting of a whitened matched filter and two variable thresholds for each symbol to be detected. The detector will make decisions on some, but most often not all, of the symbols in a transmitted sequence, and those decisions will be the same decisions as the maximum likelihood sequence detector (MLSD) would have made. The number of symbols that are detected is stochastic, varying from sequence to sequence. The basis of the detector is a bound on a cross-product of the quadratic form in the loglikelihood function for the transmitted sequence.
\end{abstract}

\section{INTRODUCTION}

Consider the detection of independent, antipodally modulated, binary symbols transmitted in blocks over a discrete-time, additive Gaussian channel with intersymbol interference (ISI). We show that by accepting that some symbols are left undetermined, it is possible to derive, in a highly computationally efficient fashion, the same decisions on individual symbols as the maximum likelihood sequence detector (MLSD) would. The proposed detector $^{1}$ is not intended as a stand-alone detector, but as a mean of extracting information in the form of MLSD-decisions out of the received data. It can for instance be used as described by Figure 1, where the decisions, together with the received signal, are fed to a complementary receiver that decides on the undetermined symbols.

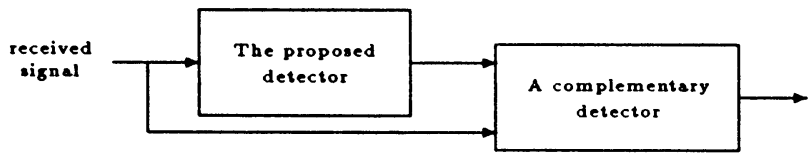

Figure 1: An example of how the detector can be used.

The detector is simple in structure and consists of a whitened matched filter and, in the case of real-valued channels and symbols, of two variable thresholds for each symbol, where the thresholds are dependent on the received signal, see Figure 2.

The detector is derived by expanding, with respect to a single symbol, the quadratic form of the log-likelihood

\footnotetext{
${ }^{1}$ This work was supported by the Swedish National Board for Industrial and Technical Development (NUTEK).
}

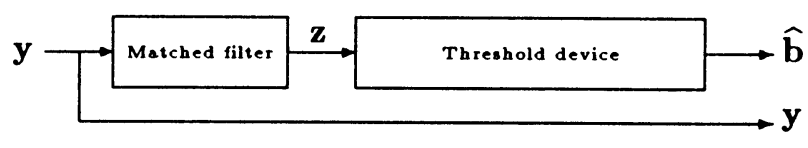

Figure 2: A block description of the proposed detector.

function for the entire transmitted sequence. This expansion leads to an expression where only one term depends on the symbol to be detected. This term is a product of that symbol and a difference between the output from a whitened matched filter and a function of the other symbols, a function that can be upper and lower bounded by two thresholds that are independent of the undetermined symbols. The symbol is detected if the corresponding output from the matched filter is either above the upper threshold or below the lower threshold. Let us assume that the transmitted symbols are binary, and consequently that the output alphabet of the detector is ternary, " +1 ", " -1 " and "don't know". With the decision on symbol number $i$ as $\widehat{b}_{i} \in\{-1,0,+1\}$, where a zero signifies "don't know", and with $m_{i, k}=\sum_{j} h_{j} h_{j+k-i}$, the thresholds can be calculated as

$$
\begin{aligned}
& \Delta_{k}^{+}:=\sum_{\left\{i \mid i \neq k, \widehat{b}_{i} \neq 0\right\}} m_{i, k} \widehat{b}_{i}+\sum_{\left\{i \mid i \neq k, \widehat{b}_{i}=0\right\}}\left|m_{i, k}\right|, \text { for all } k \\
& \Delta_{k}^{-}:=\sum_{\left\{i \mid i \neq k, \widehat{b}_{i} \neq 0\right\}} m_{i, k} \widehat{b}_{i}-\sum_{\left\{i \mid i \neq k, \widehat{b}_{i}=0\right\}}\left|m_{i, k}\right|, \text { for all } k
\end{aligned}
$$

If the output of the matched filter corresponding to bit $k$ is above $\Delta_{k}^{+}$, then a decision is made that bit $k$ is equal to one. If the output of the matched filter corresponding to bit $k$ is below $\Delta_{k}^{+}$, then bit $k$ is decided to be equal to minus one. After that some symbols have been detected new, and tighter, thresholds can be calculated and more symbols may be detected. All symbols that are detected by this procedure are detected with the MLSD-criterion, because they maximize the log-likelihood function for the transmitted sequence.

The potential of the proposed detector is dependent on its ability to make decisions. The probability of decision depends on the impulse response of the channel through equations (1) and (2). Simulations and preliminary analyses indicate that the probability of decision will be high enough in many applications for the detector to significantly improve system performance. The resulting performance will be in the gap between the performance of a complementary detector and the performance of the MLSD. 